will accept a wide range of input frequencies, can supply a wide range of sampling frequencies and will attain a significant enhancement of signal-to-noise ratio. It has been applied quite successfully in our laboratory to determine phase responses of psychophysical systems where noise corruption of the output signal is unavoidable, and where immediate knowledge of this response is needed in order to continue the experiment properly. Only fixed-point, single-precision arithmetic is used, and the program is written in PAL III. Division is accomplished by successive CLL and RAR commands. An example of the output of the algorithm is shown in Fig. 3. The response in this case is body sway in a standing human induced by a low-frequency sinusoidal current stimulus applied to the skull.

\section{REFERENCE}

RHYNE, V. T. A comparison of coherent averaging techniques for repetitive biological signals. Medical Research Engineering, August-September 1969

\title{
Simulation of neural sets
}

\author{
BRAD COX* \\ University of Chicago, Chicago, Illinois 60637
}

A program has been written for the PDP-8/I computer which simulates the behavior of 64 model neurons which may be connected together to form a functional neural net. The simulation includes provisions for studying the possible mechanisms of learning in real systems. Operation of the net requires inputs from either a robot or a robot simulator program and output from the net serves as input to such a robot, so that the entire system investigates the behavior of an organism and its brain in interaction with an environment.

\section{INTRODUCTION}

Mankind has probably been involved in contemplating the paradox of "pondering the ponderer" ever since he first possessed a brain to ponder itself with, but, while there has been some progress in unraveling the functioning of some of the brain's parts, we are still not much closer to understanding the really basic question of how it learns. Part of the problem is due to the fact that neurons are very small, and the parts of the neurons where one expects the most important aspects of their behavior to be carried out (in the synapses and dendrites) are far too small to allow measurements of their activities to be made without damaging them. It is possible to make electrodes small enough to record electrical potential changes from individual cell bodies, but we have little assurance that these are not incidental byproducts of more important events taking place at the molecular level for which we lack the tools to observed directly.

In addition, while technology has been of great help in developing tools to use in experimental brain studies, the concepts that one tends to use when thinking of present-day machinery has little applicability to biological systems. Brains have been likened to hydraulic systems, telephone exchanges, holograms, and computers, but the fact remains that

*I would like to express my appreciation to Professor Anthony Robertson and to the Committee on Mathematical Biology, for their support and interest, and to the U.S. Public Health Service for providing financial aid under Grant 1 TOI GM 2037. they operate on entirely different principles, for which we lack any good analogs.

There are, however, several characteristics of biological nerve nets which should be of help in understanding how they function. Organisms develop from single cells by following certain rules for development which eventually lead to the creation of sense organs, nervous systems, effector organs, and a body which houses these elements and maintains a fairly constant spatial relationship between them. There is every reason to believe that the brain cannot be studied in isolation from these relationships, and that the feedback loop between effector organs and the sensory apparatus is essential in maintaining reasonable responses to environmental changes and, therefore, normal brain activity.

In addition, as one examines a range of organisms, from those with very simple nervous systems and very stereotyped modes of behavior to those with millions of neurons, one finds that the apparent randomness of connections between individual neurons increases drastically. For example, the sea hare (Aplysia) has a nervous system composed of relatively few neurons which are readily identifiable from individual to individual, with each neuron always connecting to the same other neurons in case after case. In contrast, in mammals, while the gross anatomy is very reproducible, connections between developing neurons appear to be guided by laws that are very general in nature and leave much opportunity for randomness. One tends to suspect that the mechanisms of learning might have evolved to remove the burden of specifying the function of individual neurons from the genetic apparatus, thus allowing larger numbers of neurons, depending on the redundant information content of the feedback loop between muscular activity and sensory changes, to establish their functional identity. The well-known peculiarities of instincts and reflexes probably represent the existence of a backbone of genetically controlled pathways which serve as a bootstrap to enable the organism to survive in his initial contacts with his environment and to keep him functioning until sufficient learned pathways have been established.

This paper describes the current progress of our efforts to develop experimental tools and ways of thinking which we hope will lead to new progress in this field. The neural simulator program is designed to be used as the nervous system for a robot, on which sensors are mounted to encode relationships between the robot and its environment and internal variables. The outputs from the simulator will be used to control servomotors which will alter these relationships. Our next step will be to select some real biological system about which enough experimental information has been published to guide the building of a robot which can reproduce the range of bodily activity relevant to that organism. Ultimately, the entire system will be used to investigate the behavioral effects, modifications of parameters, and even the logical structure of the model neurons described in this paper.

\section{GENERAL SIMULATION LOGIC}

The current version of the simulator is operating on a PDP- $8 / \mathbb{I}$ computer with $8 \mathrm{~K}$ of memory, extended arithmetic element, and the AXO8 laboratory peripheral unit which is used for oscilloscope control. Up to 64 model neurons can be simulated, each of which can have up to 8 inputs and 8 outputs, which may be connected to any of the other neurons in the net. In addition, interface routines are 


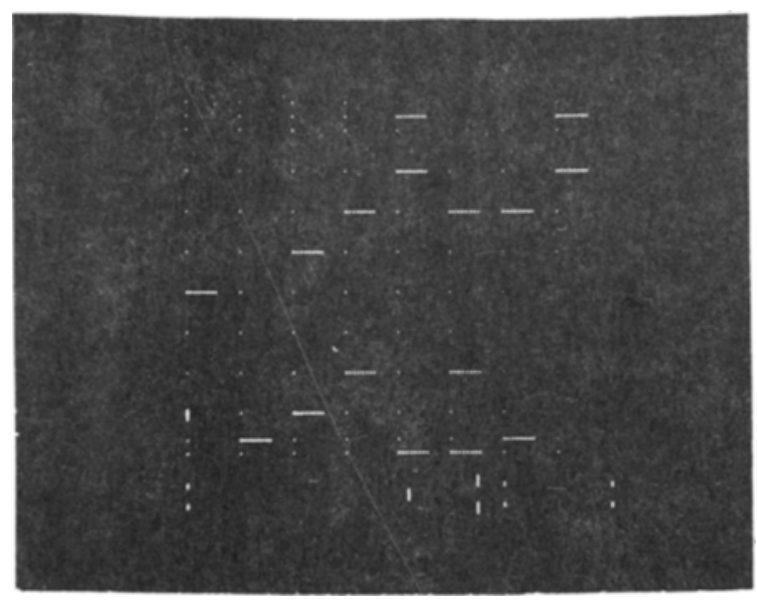

Fig. 1. Display with marker dots. See text for explanation.

available to connect up to 16 inputs and 16 outputs to external analog devices and perform the necessary conversions between analog signals and the frequency-modulated pulse trains characteristic of neural activities. These interface routines thus serve to simulate sense organs and effector organs (muscles or glands) and require very similar programming logic to that which will be described for the neuron simulation routine.

Each unit in the net is assigned an array ( 32 words for neurons, 8 words for sensors, and 16 words for muscles) which serves to specify the unit's structure (as set up by the operator during command mode) and its current status (which is determined by state variables stored in the array which depend on its history during simulation mode). Simulation of any unit's behavior involves the action of the simulation routine for that unit's type (sensor, neuron, or muscle) upon the status array for that unit. In general, successive time intervals are simulated one after the other in rapid succession so that time is quantized into intervals whose size depends on the speed of the simulation programs. Within any time interval, the sequence of operations is: (1) Read analog voltages from transducers mounted on the robot via the A-D converters. (2) Simulate sensors connected to each input channel. This involves determining whether or not each sensor will fire during this time interval by use of a counter which is recomputed each time the sensor fires from the value of the analog signal. (3) Simulate each neuron in the net. Special provisions are made so that each neuron in the net is temporally equivalent in spite of serial simulation of the neurons. (4) Simulate the muscles. Each muscle can receive inputs from up to four neurons. Firing of an input neuron causes a synapse

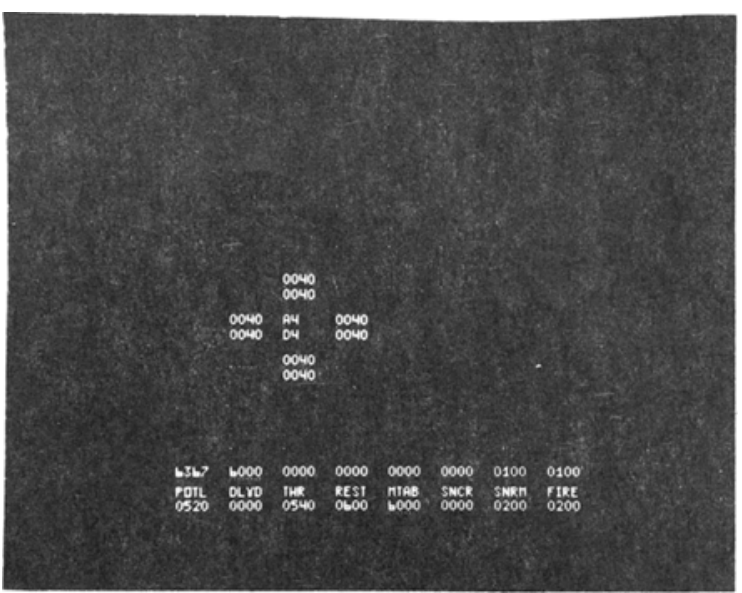

Fig. 2. Sample photograph taken from the display.

weight to be added to the current length of the muscle, the effects of which are opposed by an elastic constraint which tends to drive the muscle length to its "resting length." (5) Output each muscle length to servomotors on the robot via the D-A converters. (6) Execute a display routine which displays the current status of the net on the oscilloscope.

During normal operation, the program loops back to Step 1, and simulations of successive time intervals follow immediately. However, it is possible to execute only Step 6 by entering "single stepping mode," thereby obtaining a snapshot picture of the net's status at some time interval. Thereafter the simulation routines are executed only once each time a special Teletype key is pressed, making it possible to single-step the time scale.

The display facilities were designed for speed of operation consistent with the requirement that the operator be able to observe the important aspects of the simulation's progress. The relevant aspects of this display (Fig. 1) are: (1) Marker dots to indicate the assigned screen position for each defined unit. The top two rows of eight dots each are the assigned positions for the 16 muscle simulators, the next eight rows are for the 64 neurons, and the last two rows are for the sensors. (2) Firing indicator marks (dotted horizontal lines) to indicate which units fired during the current time interval. (3) A movable curser (a short vertical line seen at the marker dot for the neuron in the lower left corner) which is movable in eight directions by means of routines selected by eight Teletype keys. (4) A graphical display of the selected unit's status, seen at the very bottom of the display. The lines at the left and the right delimit the range allowed for the unit's state variable (membrane potential for neurons; its range is restricted to 0000 to 0777 , octal) and the short unbroken line indicates the varying position of the variable for that unit. The longer and shorter broken lines indicate the positions of the threshold and resting potential, respectively. (5) An optional "probe" routine (not seen in this picture), which the operator can add or delete at will and which displays the values of the synapse weights to the selected unit in a manner similar to that which will be described for the command mode displays.

\section{MODEL NEURON \\ CHARACTERISTICS}

This version of the simulator is set up to investigate the information processing capabilities of model neurons whose only integrative variable is the membrane potential. There are no facilities for allowing variable transmission time between units, such as exists in real neurons, because of the finite speed of signal propagation along axons, nor has any attempt been made to simulate the cable properties of dendrites, mainly because of the lack of any clear understanding of their role in neural behavior.

Each neuron is described in the computer by the contents of its "neuron status array," which is initialized by the operator during command mode and has the format shown in Table 1.

Simulation of a neuron involves the action of the "neuron simulation routine" upon the status array for that neuron. Neurons are selected for simulation from a table of addresses of neuron status arrays called the "neuron definition table," which is set up by use of command mode facilities and allows use of less than the full complement of neurons without having to spend execution time 
Table 1

\begin{tabular}{|c|c|c|}
\hline 00 & POTL & Membrane potential \\
\hline 01 & DLYD & Delayed potential \\
\hline 02 & THR & Threshold \\
\hline 03 & REST & Resting potential \\
\hline 04 & MTAB & $\begin{array}{l}\text { Metabolic time con- } \\
\text { stant and counter }\end{array}$ \\
\hline $\mathbf{0 5}$ & SNCR & Synaptic increment \\
\hline 06 & SNRM & $\begin{array}{l}\text { Synaptic normaliza- } \\
\text { tion constant }\end{array}$ \\
\hline 07 & FIRE & $\begin{array}{l}\text { Postfiring potential } \\
\text { reset value }\end{array}$ \\
\hline
\end{tabular}

10

11

12

13

14

15

16

17

20

21

22

23

24

25

26

27

30

31

32

33

34

35

36

37

Table for up to eight addresses of status arrays of input cells. The last five bits in each word are available for storage of indicators of each input cell's firing history.

Synaptic weights for the above eight input synapses. Positive numbers indicate excitatory synapses; negative numbers indicate inhibitory synapses. The excitatory synapse weights are normalized to the synaptic normalization constant.

Addresses of the synapse weights that will be used to modify the output cells' membra ne potentials in the event that this cell fires. The addresses of the output cells' status array origins are obtainable from these addresses by masking.

simulating unused neurons. This table is kept in ascending order of addresses so that the temporal equivalence logic can determine whether or not a neuron has already been simulated during the current time interval by means of a simple address comparison.

At the time that a neuron is to be simulated, its membrane potential has a value set by preceding simulations of its input cells and the first operation necessary is the comparison of the membrane potential with threshold.

If the membrane potential is less than threshold, the cell does not fire. At this time, the membrane potential is modified by use of the differential form of an exponential decay formula:

POTL

$=$ POTL + (REST - POTL $) /$ CONS

where CONS is analogous to the time constant for the decay. In the event that POTL is close to REST, the use of fixed-point arithmetic for the division gives a zero quotient, so special facilities are used to store the reciprocal of the above ratio as a counter. When this counts out, a small change is made in POTL so that it continues to exhibit exponential decay. Notice that if REST is specified to lie above THR, the neuron will exhibit spontaneous activity at a frequency determined by the time constant for the decay. The time constant and the time interval counter share the MTAB word in the status array.

If the membrane potential exceeds threshold, this indicates that the neuron is to fire and a flag is set accordingly. The refractory period is simulated by resetting the membrane potential to a low value, FIRE, after which extra excitation will be required to cause the neuron to fire again.

The next stages are executed for both cases (so that execution time does not depend on how many neurons fired per time instant and so that the synapse weights are kept normalized) and involves scanning the firing history bits in the input table to determine which of the inputs fired during the previous time interval. If this cell fired, and if a particular input cell fired, and if the synapse weight for that cell is positive, then that synapse weight is increased by the synaptic increment, SNCR. The sum of the excitatory synapse weights is also computed during this loop for use in the normalization.

Normalization involves multiplying each excitatory weight by SNCR/SUM, thus insuring that their sum remains equal to SNCR. In this way, if some input weight increases due to correlated firing, all the others decrease proportionally. Notice that this procedure does not vary inhibitory synapse weights, mainly because of the lack of a satisfactory theory for variation of inhibition. Also, this procedure tends to enhance the effects of one cell on another if they exhibit correlated activity, but I am convinced that the procedure just described is not sufficient because it lacks any provision for removing the restriction that they must fire in successive time intervals. Some degree of temporal integration might help reduce the problems we have had with synapse variations.

Finally, according to whether or not this neuron fired, modification of the status arrays of its output cells takes place. Each address in its output table is used to obtain a synapse weight This address is easily converted into the address of the membrane potential for the output neuron and to the address to be used for the storage of the firing history of this neuron. Both of these parameters must be treated in accord with the requirement that each neuron in the net be temporally equivalent and not depend on its place in the serial execution of the simulation. This requires that all transmissions between neurons need one time interval to propagate, and requires the use of the delayed potential word (DLYD) and some extra bits in the firing history table.

The final operation necessary is the construction of an extra firing history table that contains the firing status of each cell in the net during the current time interval. This table is used by the simulation mode display routines to determine which neurons fired and by the muscle simulator routines to enable interfacing neurons to muscles without requiring special provisions for their slightly different connection requirements.

\section{PROVISIONS FOR SIMULATION} CONTROL-COMMAND MODE

The importance of a well-organized and convenient procedure for program control is evident when it is realized that there are 64 neurons, 16 sensors, and 16 muscles, whose status arrays alone occupy 2,432 words of storage, each word of which must be under direct operator control. Preliminary versions of the simulator used conventional means of inputting data (paper tape), but it was quickly realized that this would never allow sufficient flexibility and convenience to make simulations feasible. For these reasons, the programs were set up so that they would receive inputs entirely according to curser control logic and make heavy use of oscilloscope displays.

The essential features of these displays are: (1) Marker dots to indicate each defined unit's screen position. Units may be defined or deleted by moving a curser to their assigned position and pressing special Teletype keys. Marker dots are not displayed for undefined units. (2) Two unit cursers which are independently movable among the assigned screen positions. The "THIS" curser is composed of a display of $\mathrm{Ax} / \mathrm{Dy}$ where $x$ and $y$ indicate the number of input and output connections, respectively, and the "THAT" curser consists of a horizontal dotted line. The position of the THIS curser selects some particular unit for parameter display and modification, and the THAT curser is used to select other units for defining connections. (3) A buffer of eight words and a curser to select some particular buffer word. The selected buffer word may be modified by use of the octal numerical Teletype keys, which enter successive digits from the right of the word. All numerical inputs are initially entered from the buffer. (4) A display of the THIS unit's parameter values, along with labels to identify them to the operator. This display also includes a movable curser which is used to select some parameter for possible replacement from the buffer. This replacement may be done for THIS unit alone, or for all units of a 
particular type in unison. (5) A display that indicates which units are currently connected to THIS unit, along with their synapse weight. Units which serve as inputs to THIS unit are indicated by having the synapse weight for their connection displayed below their marker dot, and units which serve as outputs for THIS unit have their synapse weight displayed below their marker dot. Notice that the connections shown in the sample photograph (Fig. 2) have THIS neuron connected to its nearest neighbors in a two-way hookup which I habitually use to test the programming (the pattern depicted for this neuron is repeated throughout the net). Connections between units are established by positioning the two unit cursers at the positions of the units to be connected and pressing one of two special Teletype keys according to whether THAT unit is to serve as input or output to THIS unit. The synapse weight for the connection is obtained from the buffer word selected by the buffer curser. A third Teletype key serves to delete unwanted connections, and still other keys are available for selecting special functions, such as for setting all synapse weights in the net to the same value and for clearing storage before defining new net setups.

\section{DISCUSSION}

Because of our lack of a method for introducing correlations between inputs and outputs for the net, it has been possible to test the model neurons' logic only to the extent of debugging the programs, with one exception. Instead of building a real robot, a very simple robot was simulated by program logic and connected up with an earlier version of the simulator (which used neurons very similar to those described here). Neurons were connected in a pattern which ultimately could control the robot in such a way that its design objectives were achieved, but when synaptic variations were introduced (by making SNCR nonzero) the performance of the robot tended to deteriorate rather rapidly. Apart from the possibility that the synaptic variation algorithm is completely unsuitable, this could be due to the fact that individual synapse weights are able to range from zero to SNRM, and one would expect that this range of variation was unreasonable. One would expect that any synaptic variations involved in learning in real neurons would not involve a range from no effect to unconditional firing, and also one would prefer that synapse weight variations be done after the neurons have shown correlated activities over a wider range of time intervals. Notice also that this algorithm makes it extremely unlikely that a synapse weight, once having become near zero, can ever increase again, making the situation where a neuron has several inputs with appreciable synapse weights an unstable one that promptly degenerates to the single input case.

The time scale for simulated organisms depends entirely on the size of the basic time interval, which is determined by the execution time for the programs necessary for one time interval simulation. This time must be much smaller than the average interval between neural firing for the simulated organism and may be regarded as the width of an action potential in real organisms. This time is currently on the order of $0.1 \mathrm{sec}$ for simulations of the full complement of 64 neurons and, considering the amount of computation that must be done during each time interval, this is pretty rapid. However, this must be reduced by at least an order of magnitude to allow simulations of this size to be done in real time.

Some improvement could be achieved by use of a computer with a word size of more than 12 bits, since many of the operations necessary are awkward and time consuming with a word of this size. An appealing solution for the future would be special-purpose computers with a section of "memory words" composed of independent integrated circuits capable of performing functions as described for the neural simulator routine. Control of such a setup could be done by a more or less conventional central processing unit capable of initializing the operands of the microprocessors and displaying their status to the operator. 